University of Wollongong

Research Online

Faculty of Engineering and Information

Faculty of Engineering and Information

Sciences - Papers: Part A

Sciences

$1-1-2008$

Single- and multi-carrier IDMA schemes with cyclic prefixing and zero padding techniques

Qinghua Guo

City University of Hong Kong, qguo@uow.edu.au

Xiaojun Yuan

Chinese University of Hong Kong

Li Ping

City University of Hong Kong

Follow this and additional works at: https://ro.uow.edu.au/eispapers

Part of the Engineering Commons, and the Science and Technology Studies Commons

Research Online is the open access institutional repository for the University of Wollongong. For further information contact the UOW Library: research-pubs@uow.edu.au 


\title{
Single- and multi-carrier IDMA schemes with cyclic prefixing and zero padding techniques
}

\author{
Abstract \\ In this paper, we investigate single-carrier (SC) and multi-carrier IDMA schemes with cyclic prefixing (CP) \\ and zero padding (ZP) techniques. These schemes are called SC-IDMA-CP, SC-IDMA-ZP, orthogonal \\ frequency-division multiplexing (OFDM)-IDMA-CP and OFDM-IDMA-ZP, respectively. By exploiting the \\ circulant property of channel matrices, the multi-user detection of these schemes can be efficiently \\ realised with per-user complexity independent of the number of users and the length of inter-symbol \\ interference (ISI) channels. We show the potential advantages of ZP-based schemes in power efficiency \\ and convergence speed. Simulation results are provided to compare the four schemes. Copyright (C) 2008 \\ John Wiley \& Sons, Ltd.

\section{Keywords} \\ padding, zero, prefixing, techniques, cyclic, single, schemes, idma, carrier, multi \\ Disciplines \\ Engineering | Science and Technology Studies

\section{Publication Details} \\ Q. Guo, X. Yuan \& L. Ping, "Single- And multi-carrier IDMA schemes with cyclic prefixing and zero padding \\ techniques," European Transactions on Telecommunications, vol. 19, (5) pp. 537-547, 2008.
}




\title{
Single- and multi-carrier IDMA schemes with cyclic prefixing and zero padding techniques
}

\author{
Qinghua Guo, Xiaojun Yuan and Li Ping* \\ Department of Electronic Engineering, City University of Hong Kong, Hong Kong SAR
}

\begin{abstract}
SUMMARY
In this paper, we investigate single-carrier (SC) and multi-carrier IDMA schemes with cyclic prefixing (CP) and zero padding (ZP) techniques. These schemes are called SC-IDMA-CP, SC-IDMA-ZP, orthogonal frequency-division multiplexing (OFDM)-IDMA-CP and OFDM-IDMA-ZP, respectively. By exploiting the circulant property of channel matrices, the multi-user detection of these schemes can be efficiently realised with per-user complexity independent of the number of users and the length of inter-symbol interference (ISI) channels. We show the potential advantages of ZP-based schemes in power efficiency and convergence speed. Simulation results are provided to compare the four schemes. Copyright (C) 2008 John Wiley \& Sons, Ltd.
\end{abstract}

\section{INTRODUCTION}

Orthogonal frequency-division multiplexing (OFDM) and single-carrier (SC) transmission with frequency domain equalisation (FDE) are two efficient techniques for combating inter-symbol interference (ISI) in communication systems [1-3]. Both OFDM and SC transmission with FDE (SC-FDE) can be realised using the cyclic prefixing (CP) technique at the cost of an extra power overhead. Recently, zero padding (ZP) has also been studied as an alternative to $\mathrm{CP}$ in both OFDM and SC systems [4-6]. The advantages of OFDM-ZP over OFDM-CP have been discussed in Reference [6] for single-user systems with non-iterative detectors. The turbo receiver studied in Reference [4] involves iterative decoding, but not iterative joint equalisation and decoding. The use of $\mathrm{ZP}$ has the following two opposite consequences: (i) ZP can avoid the power overhead associated with CP, but (ii) OFDM-ZP cannot maintain the orthogonality among sub-carriers as OFDM-CP does, which results in an interference problem. Hence, with conventional noniterative detection methods, these two effects may offset each other and so the potential of $\mathrm{ZP}$ is somewhat unclear.

In this paper, we investigate both CP- and ZP-based multi-user systems with iterative detectors. This work is motivated by two recent developments in wireless communication systems:

(i) Joint iterative signal estimation and decoding [7, 8] has emerged as an efficient solution to the interference problem in communication systems. This provides a promising means to resolve the interference problem for the ZP technique.

(ii) It has been shown that significant multi-user gain is achievable by allowing concurrent multi-user transmission at transmitters and employing multiuser detection at receivers [9]. The cost of multi-user detection is a serious issue in ISI channels. Even the rake-type receiver (for both code-division-multipleaccess (CDMA) and interleave-division-multipleaccess (IDMA)) has complexity $O(L+1)$ where $L$ is the channel memory length. When $L$ is very large, the complexity can be a concern. It is therefore of interest to investigate low-cost solutions for such multi-user systems in severe ISI channels.

In this paper, we apply the $\mathrm{CP}$ and $\mathrm{ZP}$ techniques to single- and multi-carrier IDMA, resulting in four schemes: SC-IDMA-CP, SC-IDMA-ZP, OFDM-IDMA-CP and OFDM-IDMA-ZP. A unified detection approach for the

*Correspondence to: Li Ping, Department of Electronic Engineering, City University of Hong Kong, Hong Kong SAR. E-mail: eeliping @ cityu.edu.hk 
four schemes is proposed. We show that the multi-user detection of these schemes can be efficiently realised with per-user complexity independent of $L$ as well as of the number of users (denoted by $K$ ), which is significantly lower than other alternatives. Simulation results are provided to compare the four schemes and demonstrate the full potential of ZP-based schemes (relative to the CP-based ones) in power efficiency and convergence speed.

Recently, there has been increasing interest in SC-FDE and OFDM techniques. For example, the 3GPP Long Term Evolution (LTE) [10] is considering SC-FDE as a candidate for uplink transmission and OFDM for downlink transmission. The work presented in this paper shows that $\mathrm{ZP}$ provides a more power efficient option than $\mathrm{CP}$.

\section{IDMA SYSTEM MODEL AND ITERATIVE DETECTION PRINCIPLES}

\subsection{IDMA system model}

Consider a multiple-access system modelled as

$$
\boldsymbol{y}=\sum_{k=1}^{K} \boldsymbol{A}_{k} \boldsymbol{x}_{k}+\boldsymbol{n}
$$

where $\boldsymbol{y}$ is the observation vector, $\boldsymbol{x}_{k}$ the transmitted signal vector of user $k, \boldsymbol{A}_{k}$ the transfer matrix for user $k, \boldsymbol{n}$ a vector of noise samples, distributed according to a circular symmetric complex Gaussian distribution with zero mean and covariance matrix $\sigma^{2} \boldsymbol{I}, \boldsymbol{I}$ an identity matrix with proper size and $K$ the number of users in the system. In Equation (1), $\boldsymbol{A}_{k}$ may include the effect of pre-coding at the transmitter, channel response and pre-processing at the receiver. If there is pre-processing at the receiver, the observation vector $\boldsymbol{y}$ may be different from the channel output vector $\boldsymbol{r}$.

IDMA [11, 12] is a multiple-access scheme in which multi-user detection can be efficiently realised. The IDMA transmitter architecture (without pre-coding) is illustrated in the upper part of Figure 1. For each user $k$, the source data $\boldsymbol{d}_{k}$ is first encoded by using a forward-error-correction (FEC) code denoted by $\boldsymbol{C}$, and then permuted by a userspecific random interleaver (denoted by $\Pi_{k}$ ). The resultant bit stream is mapped to a complex signal stream by using quadrature phase shift keying (QPSK) with Gray mapping. This produces the signal vector $\boldsymbol{x}_{k}$ for transmission over the ISI channel characterised by the complex channel matrix $\boldsymbol{A}_{k}$.

Copyright () 2008 John Wiley \& Sons, Ltd.

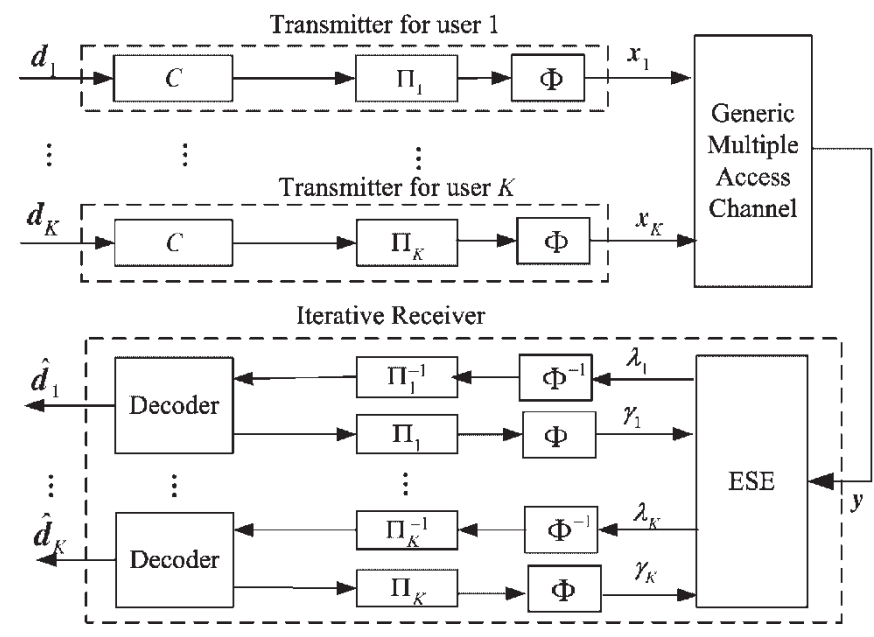

Figure 1. The generic IDMA transmitter and (iterative) receiver. $\Pi_{k}$ denotes the interleaver for user $k$ and $\Pi_{k}^{-1}$ the corresponding de-interleaver. $\Phi$ and $\Phi^{-1}$ denote QPSK mapper and de-mapper, respectively.

\subsection{Iterative detection principles}

The iterative receiver is shown in the lower part of Figure 1. Let $x_{k}(i)$ be the $i$ th entry of $\boldsymbol{x}_{k}$. The elementary signal estimator (ESE) computes the extrinsic log-likelihood ratio (LLR) for the two bits carried by $x_{k}(i)$ as

$$
\begin{aligned}
& \lambda_{k}^{\mathrm{Re}}(i) \equiv \ln \frac{p\left(\boldsymbol{y} \mid x_{k}^{\mathrm{Re}}(i)=+1\right)}{p\left(\boldsymbol{y} \mid x_{k}^{\mathrm{Re}}(i)=-1\right)} \\
& \lambda_{k}^{\operatorname{Im}}(i) \equiv \ln \frac{p\left(\boldsymbol{y} \mid x_{k}^{\operatorname{Im}}(i)=+1\right)}{p\left(\boldsymbol{y} \mid x_{k}^{\operatorname{Im}}(i)=-1\right)}
\end{aligned}
$$

respectively, or in a compact complex form as

$$
\lambda_{k}(i) \equiv \lambda_{k}^{\operatorname{Re}}(i)+j \lambda_{k}^{\operatorname{Im}}(i), \forall k, i
$$

with the FEC coding constraint ignored, that is the ESE operates as if $\boldsymbol{x}_{k}$ contains uncoded bits. In the above equations, the superscripts 'Re' and 'Im' denote real and imaginary parts, respectively. We rewrite $\left\{\lambda_{k}(i), \forall i\right\}$ in a vector form

$$
\lambda_{k} \equiv\left[\lambda_{k}(0), \ldots, \lambda_{k}(i), \ldots\right]^{T}
$$

where the superscript ' $T$ ' denotes the transpose operation. For each user $k$, the elements of sequence $\lambda_{k}$ are de-mapped to produce a real LLR sequence that is used, after deinterleaving, as the inputs to the a posteriori probability 
(APP) decoder. The decoder output sequence (containing the extrinsic LLRs) is then interleaved and mapped to a stream of complex LLRs $\gamma_{k} \equiv\left[\gamma_{k}(0), \ldots, \gamma_{k}(i), \ldots\right]^{T}$ with $i$ th element defined as

$$
\gamma_{k}(i) \equiv \gamma_{k}^{\operatorname{Re}}(i)+j \gamma_{k}^{\operatorname{Im}}(i)
$$

where

$$
\begin{aligned}
\gamma_{k}^{\mathrm{Re}}(i) & \equiv \ln \frac{p\left(x_{k}^{\mathrm{Re}}(i)=+1\right)}{p\left(x_{k}^{\mathrm{Re}}(i)=-1\right)} \\
\gamma_{k}^{\operatorname{Im}}(i) & \equiv \ln \frac{p\left(x_{k}^{\operatorname{Im}}(i)=+1\right)}{p\left(x_{k}^{\operatorname{Im}}(i)=-1\right)}
\end{aligned}
$$

After decoding, the ESE refines the estimates in Equation (2) using the feedback $\gamma_{k}$. This iterative process continues until the algorithm converges. Hard decisions are made in the final iteration to produce the estimates $\left\{\hat{\boldsymbol{d}}_{k}\right\}$. The detailed discussions can be found in References [7, 8, 11, 12]. The DEC follows the standard APP decoding principle [13]. In what follows, we consider the realisation of the ESE.

\subsection{The ESE function}

The derivation for the ESE function based on a joint Gaussian assumption has been given in References [14] and [15]. It can be extended to a complex system with QPSK signalling as follows. We assume that the channel state information is available at the receiver. The ESE computes the extrinsic LLRs defined in Equation (2) based on the channel state information, the observation vector $\boldsymbol{y}$ and the a priori means and variances of $\left\{x_{k}^{R e}(i)\right.$ and $\left.x_{k}^{I m}(i), \forall k, i\right\}$ that can be calculated using the feedback information from the decoders, that is for each QPSK symbol $x_{k}(i)$,

$$
\begin{aligned}
\mathrm{E}\left(x_{k}^{\mathrm{Re}}(i)\right) & =\tanh \left(\gamma_{k}^{\mathrm{Re}}(i) / 2\right) \\
\mathrm{E}\left(x_{k}^{\operatorname{Im}}(i)\right) & =\tanh \left(\gamma_{k}^{\operatorname{Im}}(i) / 2\right) \\
v_{k}(i) & =\underbrace{\left.1-\left(x_{k}^{\mathrm{Re}}(i)\right)\right)^{2}}_{v_{x_{k}^{\mathrm{Re}}}(i)}+\underbrace{1-\left(x_{i}\right.}_{v_{x_{k}^{\operatorname{Im}}}}
\end{aligned}
$$

where $\mathrm{E}(\cdot)$ denotes the expectation operation. Let $\mathrm{E}\left(\boldsymbol{x}_{\mathrm{k}}\right)=\mathrm{E}\left(\boldsymbol{x}_{\mathrm{k}}^{\mathrm{Re}}\right)+j \mathrm{E}\left(\boldsymbol{x}_{\mathrm{k}}^{\mathrm{Im}}\right)$ and $\boldsymbol{V}_{k}$ be the a priori mean vector and covariance matrix of $\boldsymbol{x}_{k}$, respectively. Due to the presence of the interleavers, we can assume that the Copyright (C) 2008 John Wiley \& Sons, Ltd. entries in $\boldsymbol{x}_{k}$ are approximately uncorrelated and hence $\boldsymbol{V}_{k}=\operatorname{diag}\left\{v_{k}(0), v_{k}(1), \ldots, v_{k}(i), \ldots\right\}, \quad$ where $\operatorname{diag}\{\cdot\}$ returns a diagonal matrix with the elements in the brace ordered on the main diagonal. To reduce complexity, we replace all $\left\{v_{k}(i), \forall i\right\}$ by their average denoted by $v_{k}$. Then

$$
\boldsymbol{V}_{k}=v_{k} \boldsymbol{I}, \forall k
$$

We further assume that the a priori uncertainty of the bits for each user are equal, that is

$$
\begin{aligned}
\operatorname{Cov}\left(\operatorname{Re}\left\{\boldsymbol{x}_{k}\right\}, \operatorname{Re}\left\{\boldsymbol{x}_{k}\right\}\right) & =\operatorname{Cov}\left(\operatorname{Im}\left\{\boldsymbol{x}_{k}\right\}, \operatorname{Im}\left\{\boldsymbol{x}_{k}\right\}\right) \\
& =2^{-1} \operatorname{Cov}\left(\boldsymbol{x}_{k}, \boldsymbol{x}_{k}\right)=2^{-1} v_{k} \boldsymbol{I}
\end{aligned}
$$

This is clearly not an optimal treatment, but numerical results show that the performance loss due to this approximation is marginal [16].

Define

$$
\begin{aligned}
\underline{\boldsymbol{x}}_{k} & =\left[\begin{array}{l}
\operatorname{Re}\left\{\boldsymbol{x}_{k}\right\} \\
\operatorname{Im}\left\{\boldsymbol{x}_{k}\right\}
\end{array}\right], \underline{\boldsymbol{y}}=\left[\begin{array}{l}
\operatorname{Re}\{\boldsymbol{y}\} \\
\operatorname{Im}\{\boldsymbol{y}\}
\end{array}\right] \\
\underline{\boldsymbol{n}} & =\left[\begin{array}{l}
\operatorname{Re}\{\boldsymbol{n}\} \\
\operatorname{Im}\{\boldsymbol{n}\}
\end{array}\right], \underline{\boldsymbol{A}}_{k}=\left[\begin{array}{ll}
\operatorname{Re}\left\{\boldsymbol{A}_{k}\right\} & -\operatorname{Im}\left\{\boldsymbol{A}_{k}\right\} \\
\operatorname{Im}\left\{\boldsymbol{A}_{k}\right\} & \operatorname{Re}\left\{\boldsymbol{A}_{k}\right\}
\end{array}\right]
\end{aligned}
$$

where the operations $\operatorname{Re}\{\cdot\}$ and $\operatorname{Im}\{\cdot\}$ denote taking real and imaginary parts, respectively. The complex model (1) is equivalent to the following real model

$$
\underline{\boldsymbol{y}}=\sum_{k=1}^{K} \underline{\boldsymbol{A}}_{k} \underline{\boldsymbol{x}}_{k}+\underline{\boldsymbol{n}}
$$

Let us focus on $\underline{x}_{k}(i)$, the $i$ th element (coded bit) in $\underline{x}_{k}$, and treat all the other bits as interference. We can rewrite Equation (11) in a signal-plus-distortion form as

$$
\underline{\boldsymbol{y}}=\underline{\boldsymbol{a}}_{k}(i) \underline{x}_{k}(i)+\underline{\xi}_{k}(i)
$$

where $\underline{\boldsymbol{a}}_{k}(i)$ is the $i$ th column of $\underline{\boldsymbol{A}}_{k}$ and

$$
\underline{\xi}_{k}(i)=\sum_{k^{\prime}=1}^{K} \underline{\boldsymbol{A}}_{k^{\prime}} \underline{\boldsymbol{x}}_{k^{\prime}}-\underline{\boldsymbol{a}}_{k}(i) \underline{x}_{k}(i)+\underline{\boldsymbol{n}}
$$

(7c)We approximate $\xi_{k}(i)$ by a joint Gaussian random variable vector (which can be justified by the central limit theorem) with covariance matrix given by

$$
\begin{aligned}
\underline{\boldsymbol{R}}_{k}(i) & \equiv \operatorname{Cov}\left(\underline{\xi}_{k}(i), \underline{\xi}_{k}(i)\right) \\
& =2^{-1}\left(\sum_{k^{\prime}=1}^{K} v_{k^{\prime}} \underline{\boldsymbol{A}}_{k^{\prime}} \underline{\boldsymbol{A}}_{k^{\prime}}^{T}-v_{k} \underline{\boldsymbol{a}}_{k}(i) \underline{\boldsymbol{a}}_{k}(i)^{T}+\sigma^{2} \boldsymbol{I}\right)
\end{aligned}
$$


Hence, the extrinsic LLR of $\underline{x}_{k}(i)$ can be calculated as

$$
\begin{aligned}
\underline{\lambda}_{k}(i) & =\ln \frac{\exp \left(-\frac{1}{2} \boldsymbol{q}_{+}^{T}\left(\underline{\boldsymbol{R}}_{k}(i)\right)^{-1} \boldsymbol{q}_{+}\right)}{\exp \left(-\frac{1}{2} \boldsymbol{q}_{-}^{T}\left(\underline{\boldsymbol{R}}_{k}(i)\right)^{-1} \boldsymbol{q}_{-}\right)} \\
& =2 \underline{\boldsymbol{a}}_{k}(i)^{T} \underline{\boldsymbol{R}}_{k}(i)^{-1}\left(\underline{\boldsymbol{y}}-\mathrm{E}(\underline{\boldsymbol{y}})+\underline{\boldsymbol{a}}_{k}(i) \mathrm{E}\left(\underline{x}_{k}(i)\right)\right) \\
& =\frac{2 \underline{\boldsymbol{a}}_{k}(i)^{T} \underline{\boldsymbol{R}}^{-1}\left(\underline{\boldsymbol{y}}-\mathrm{E}(\underline{\boldsymbol{y}})+\underline{\boldsymbol{a}}_{k}(i) \mathrm{E}\left(\underline{x}_{k}(i)\right)\right)}{1-2^{-1} v_{k} \underline{\boldsymbol{a}}_{k}(i)^{T} \underline{\boldsymbol{R}}^{-1} \underline{\boldsymbol{a}}_{k}(i)}
\end{aligned}
$$

where

$$
\begin{aligned}
\boldsymbol{q}_{+} & =\left(\underline{\boldsymbol{y}}-\underline{\boldsymbol{a}}_{k}(i)-\mathrm{E}\left(\underline{\xi}_{k}(i)\right)\right. \\
\boldsymbol{q}_{-} & =\left(\underline{\boldsymbol{y}}+\underline{\boldsymbol{a}}_{k}(i)-\mathrm{E}\left(\underline{\xi}_{k}(i)\right)\right. \\
\underline{\boldsymbol{R}} & =2^{-1}\left(\sum_{k^{\prime}=1}^{K} v_{k^{\prime}} \underline{\boldsymbol{A}}_{k^{\prime}} \underline{\boldsymbol{A}}_{k^{\prime}}^{T}+\sigma^{2} \boldsymbol{I}\right)
\end{aligned}
$$

and the derivation of Equation (15b) from Equation (15a) is based on the matrix inversion lemma [17]. Define

$$
\boldsymbol{R} \equiv \operatorname{Cov}(\boldsymbol{y}, \boldsymbol{y})=\sum_{k=1}^{K} v_{k} \boldsymbol{A}_{k} \boldsymbol{A}_{k}^{H}+\sigma^{2} \boldsymbol{I}
$$

where the superscript ' $H$ ' denotes the conjugate transpose. Use $\boldsymbol{a}_{k}(i)$ to denote the $i$ th column of $\boldsymbol{A}_{k}$. We can verify that

$$
\lambda_{k}(i)=\frac{4 \boldsymbol{a}_{k}(i)^{H} \boldsymbol{R}^{-1}\left(\boldsymbol{y}-\mathrm{E}(\boldsymbol{y})+\boldsymbol{a}_{k}(i) \mathrm{E}\left(x_{k}(i)\right)\right)}{1-v_{k} \boldsymbol{a}_{k}(i)^{H} \boldsymbol{R}^{-1} \boldsymbol{a}_{k}(i)}
$$

based on Equations (3) and (15b) and the fact that, for an invertible complex matrix $\boldsymbol{B}$, the inverse of matrix $\underline{\boldsymbol{B}}$ defined as

$$
\underline{\boldsymbol{B}} \equiv\left[\begin{array}{cc}
\operatorname{Re}\{\boldsymbol{B}\} & -\operatorname{Im}\{\boldsymbol{B}\} \\
\operatorname{Im}\{\boldsymbol{B}\} & \operatorname{Re}\{\boldsymbol{B}\}
\end{array}\right]
$$

is given by

$$
\underline{\boldsymbol{B}}^{-1}=\left[\begin{array}{cc}
\operatorname{Re}\left\{\boldsymbol{B}^{-1}\right\} & -\operatorname{Im}\left\{\boldsymbol{B}^{-1}\right\} \\
\operatorname{Im}\left\{\boldsymbol{B}^{-1}\right\} & \operatorname{Re}\left\{\boldsymbol{B}^{-1}\right\}
\end{array}\right]
$$

We can rewrite Equation (18) as

$$
\begin{aligned}
\lambda_{k}(i)= & 4 \frac{\boldsymbol{a}_{k}(i)^{H} \boldsymbol{R}^{-1}\left(\boldsymbol{y}-\sum_{k^{\prime}=1}^{K} \boldsymbol{A}_{k^{\prime}} E\left(\boldsymbol{x}_{k^{\prime}}\right)\right)}{1-v_{k} \boldsymbol{a}_{k}(i)^{H} \boldsymbol{R}^{-1} \boldsymbol{a}_{k}(i)} \\
& +4 \frac{\boldsymbol{a}_{k}(i)^{H} \boldsymbol{R}^{-1} \boldsymbol{a}_{k}(i) E\left(x_{k}(i)\right)}{1-v_{k} \boldsymbol{a}_{k}(i)^{H} \boldsymbol{R}^{-1} \boldsymbol{a}_{k}(i)}
\end{aligned}
$$

Copyright (C) 2008 John Wiley \& Sons, Ltd.
Define

$$
\boldsymbol{U}_{k}=\left(\boldsymbol{A}_{k}^{H} \boldsymbol{R}^{-1} \boldsymbol{A}_{k}\right)_{\mathrm{diag}}
$$

where $(\cdot)_{\text {diag }}$ represents a diagonal matrix that only contains the main diagonal part of the matrix in the parentheses. We can then express the ESE function in a concise vector form as

$$
\begin{aligned}
\lambda_{k}= & 4\left(I-v_{k} \boldsymbol{U}_{k}\right)^{-1} \\
& \left(\boldsymbol{A}_{k}^{H} \boldsymbol{R}^{-1}\left(\boldsymbol{y}-\sum_{k^{\prime}=1}^{K} \boldsymbol{A}_{k^{\prime}} E\left(\boldsymbol{x}_{k^{\prime}}\right)\right)+\boldsymbol{U}_{k} E\left(\boldsymbol{x}_{k}\right)\right)
\end{aligned}
$$

Note that $\gamma_{k}$ and $\lambda_{k}$, respectively, correspond to the input and output of user $k$ in the ESE module in Figure 1. The feedback variables $\left\{\gamma_{k}\right\}$ in Figure 1 are used to compute $\left\{v_{k}\right\}$ and $\left\{\mathrm{E}\left(\boldsymbol{x}_{\mathrm{k}}\right)\right\}$ in Equation (23) according to Equations (7) and (8). The most computational intensive operation in Equation (23) is related to calculating $\boldsymbol{R}^{-1}$ and $\boldsymbol{U}_{k}$. In Sections 3 and 4, we will discuss computationally efficient techniques for calculating these quantities.

\section{SC-IDMA SYSTEMS}

We first discuss transmission techniques in Subsections 3.1 and 3.2. These techniques facilitate the fast Fourier transform (FFT)-based fast implementation techniques to be discussed in Subsection 3.3 for the detector in Equation (23).

\subsection{SC-IDMA-CP}

Consider a multi-access system, in which we assume the users are approximately synchronised, that is the synchronisation error between users is small so that it can be treated together with multi-path delay. We also assume the channel is quasi-static within one transmitted block.

The use of CP can avoid inter-block interference (IBI) and converts linear convolution to cyclic convolution $[1,2]$, which greatly facilitates detection at receiver. More specifically, for IDMA-CP [21], a cyclic prefix is added to $\boldsymbol{x}_{k}$ (of length $J$ ), that is the actual transmitted signal vector becomes $\boldsymbol{x}_{k}^{T}=\left[\underline{\boldsymbol{p}}_{k}^{T} \boldsymbol{x}_{k}^{T}\right]$, where $\underline{\boldsymbol{p}}_{k}^{T}$ is a copy of the last $L$ entries of $\boldsymbol{x}_{k}$. At receiver side, after the removal of the cyclic prefix, the received signal can be represented as

$$
\boldsymbol{y}=\sum_{k=1}^{K} \boldsymbol{H}_{k}^{\mathrm{cp}} \boldsymbol{x}_{k}+\boldsymbol{n}
$$


where each $\boldsymbol{H}_{k}^{\mathrm{cp}}$ is a circulant matrix with the first column given by $\boldsymbol{h}_{k} \equiv\left[h_{k}(0), h_{k}(1), h_{k}(L), 0, \ldots, 0\right]^{T}$ ( $\left[h_{k}(0), h_{k}(1), \ldots, h_{k}(L)\right]$ denotes the channel taps of user $k)$. The $i$ th column of $\boldsymbol{H}_{k}^{\mathrm{cp}}$ is a cyclic shift of its first column by $i$ positions. An example of $\boldsymbol{H}_{k}^{\mathrm{cp}}$ (with $L=2$ ) can be found in Figure 2. (The matrix labelled by ' $\boldsymbol{H}$ '

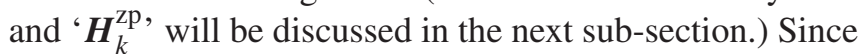
Equation (24) is a special case of Equation (1), the result (23) can be directly applied.

\subsection{SC-IDMA-ZP}

In the ZP technique, a string of zeros (denoted by $\mathbf{0}$ ) is appended after $\boldsymbol{x}_{k}$. The length of the zero string should be larger than the channel memory length $L$ to avoid IBI. Interestingly, ZP can also be used to transform the channel matrix to a circulant matrix. Potentially, the ZP technique can save the extra energy consumed by cyclic prefixes since the transmitter can keep silent during the zero interval. In the ZP technique, the received signal can be expressed as

$$
\boldsymbol{y}=\sum_{k=1}^{K}\left[\begin{array}{ll}
\boldsymbol{H}_{k} & \tilde{\boldsymbol{H}}_{k}
\end{array}\right]\left[\begin{array}{c}
\boldsymbol{x}_{k} \\
\mathbf{0}
\end{array}\right]+\boldsymbol{n}
$$

The trick here is that we can freely alter the channel matrix part $\tilde{\boldsymbol{H}}_{k}$ corresponding to $\mathbf{0}$ without affecting $\boldsymbol{y}$. We select $\tilde{\boldsymbol{H}}_{k}$ to make $\boldsymbol{H}_{k}^{\text {zp }}$ circulant, as shown in Figure 2, in order to apply the FFT technique discussed in Subsection 3.3. In this case, we can rewrite Equation (25) as

$$
\boldsymbol{y}=\sum_{k=1}^{K} \boldsymbol{H}_{k}^{\mathrm{zp}}\left[\begin{array}{c}
\boldsymbol{x}_{k} \\
\mathbf{0}
\end{array}\right]+\boldsymbol{n}
$$

Note that the size of $\boldsymbol{H}_{k}^{\mathrm{zp}}$ is expanded to $(J+L) \times(J+L)$.
Figure 3 shows the multi-paths for user $k$ in the ZP and $\mathrm{CP}$ techniques. The received signal is the superposition of these multi-paths for all the users. From Figure 3 (and also by comparing Equation (26) with Equation (24)), we can see that $\mathrm{ZP}$ can be regarded as $\mathrm{CP}$ with zero prefixes. However, with $\mathrm{CP}$, the cyclic prefix part is discarded at the receiver and so the received signal is not fully utilised. With ZP, on the other hand, the received power is fully utilised. This can also be seen from Figure 3. The observation window of $y$ in the $\mathrm{CP}$ technique is narrower than the energy spreading range of $\boldsymbol{x}_{k}$ and its cyclic prefix. On the other hand, the observation window of $\boldsymbol{y}$ in the ZP technique coincides with the energy spreading range of $\boldsymbol{x}_{k}$. This comparison indicates the advantage of ZP.

It is also seen from Figure 3 that a signal block $\boldsymbol{x}_{k}$ is extended during the transmission process due to the multipath effect. The detection in a ZP system is based on the entire energy spreading range of $\boldsymbol{x}_{k}$, which is larger than the transmitted block length of $\boldsymbol{x}_{k}$. This introduces an extra spreading factor. Therefore, ZP enjoys an additional spreading gain. We will show this using simulation results in Section 5.

\subsection{The ESE function of SC-IDMA}

Now consider the receiver. Both the CP scheme (24) and ZP scheme (26) can be treated as special cases of Equation (1). Hence, the ESE function (23) can be applied to both cases when realising the ESE. An important common trait of the above two schemes is that both $\boldsymbol{H}_{k}^{\mathrm{cp}}$ and $\boldsymbol{H}_{k}^{\mathrm{zp}}$, for $\forall k$, are circulant, based on which fast implementation of the ESE can be realised.

For notational convenience, our discussion below will be based on the unified circulant channel matrix $\boldsymbol{A}_{k}$. We assume that $\boldsymbol{A}_{k}$ is an $N \times N$ matrix $(N=$

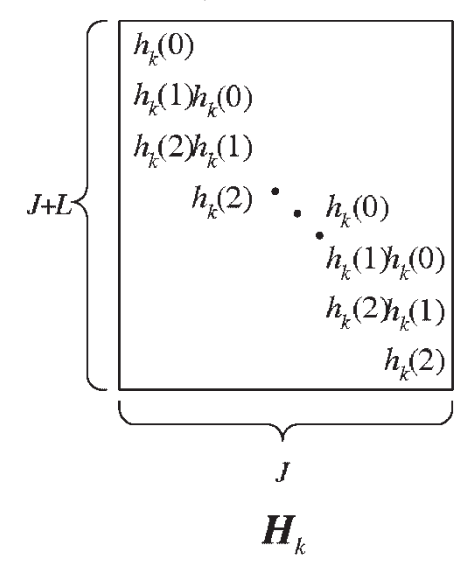

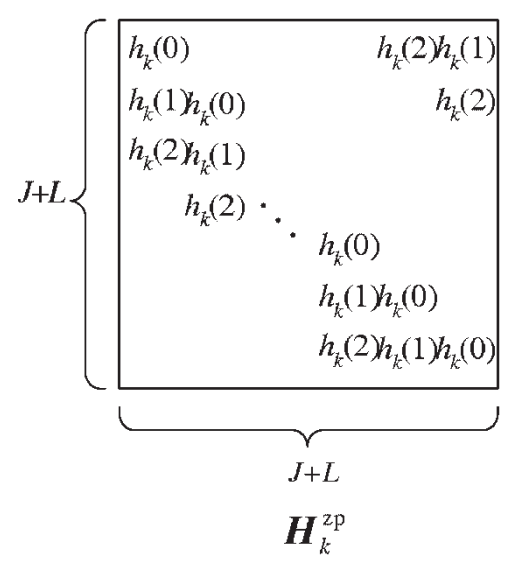

Figure 2. An example of the channel transfer matrices of user $k(L=2)$. 


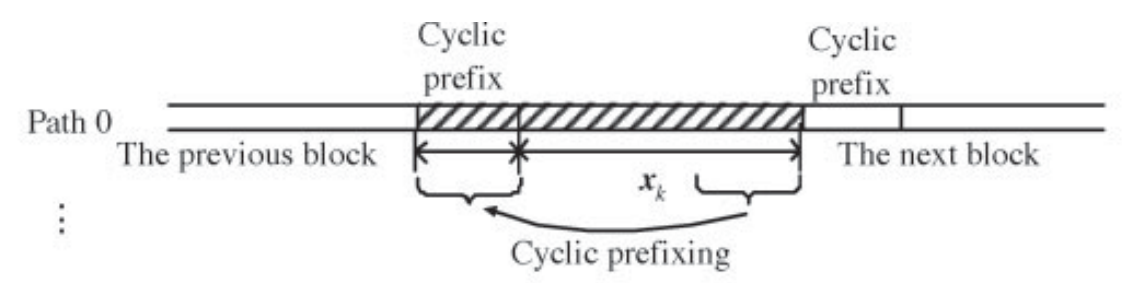

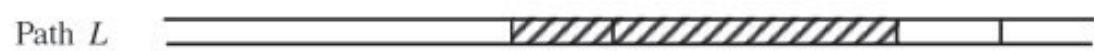

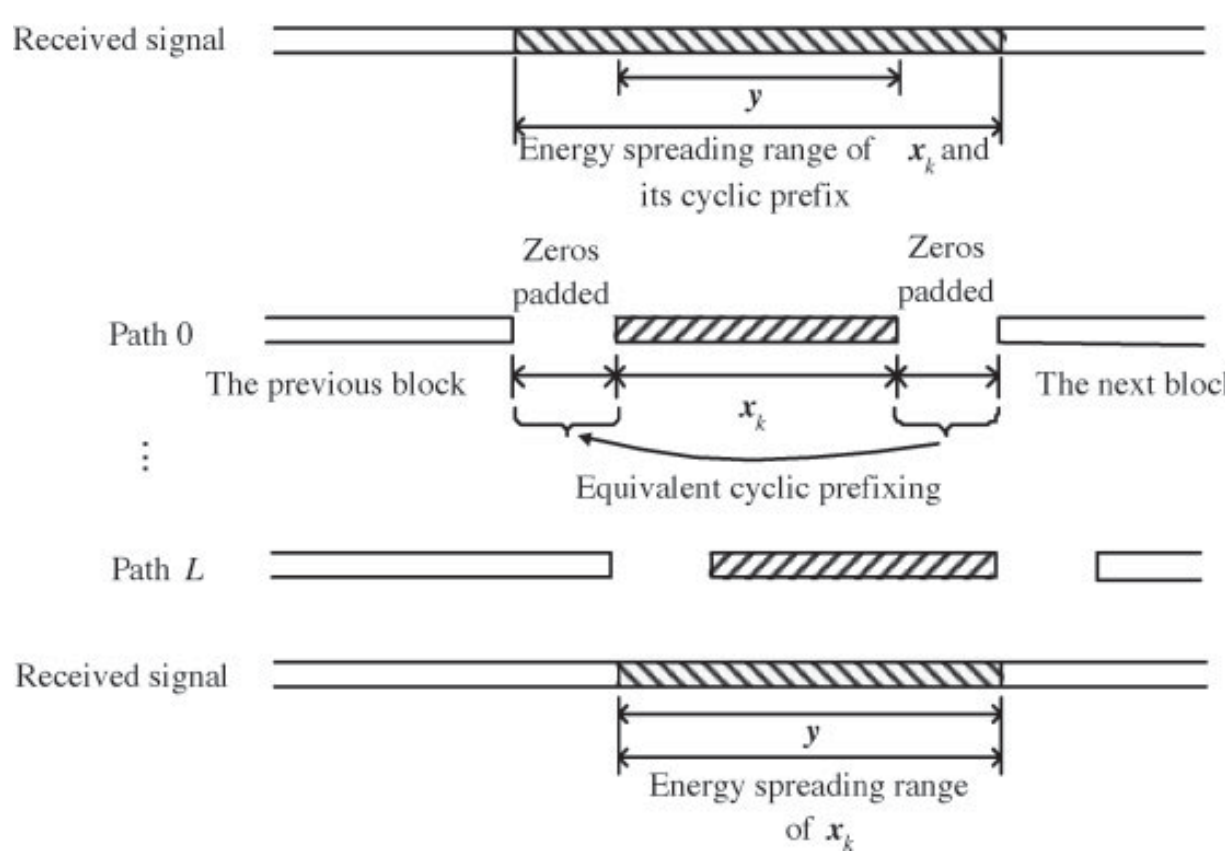

Figure 3. Comparison of the $\mathrm{CP}$ and $\mathrm{ZP}$ techniques.

$J$ when $\boldsymbol{A}_{k}$ represents $\boldsymbol{H}_{k}^{\mathrm{cp}}$ and $N=J+L$ when $\boldsymbol{A}_{k}$ represents $\boldsymbol{H}_{k}^{\mathrm{zp}}$ ). Let $\boldsymbol{F}$ be the normalised discrete Fourier transform (DFT) matrix with the $\left(i, i^{\prime}\right)$-th entry given by $N^{-1 / 2} \exp \left(-j 2 \pi i i^{\prime} / N\right)$. Clearly $\boldsymbol{F} \boldsymbol{F}^{H}=\boldsymbol{I}$. It is well known that a circulant matrix can be diagonalised by DFT and IDFT matrices as follows

$$
\begin{aligned}
\boldsymbol{G}_{k} & =\boldsymbol{F} \boldsymbol{A}_{k} \boldsymbol{F}^{H} \\
\boldsymbol{A}_{k} & =\boldsymbol{F}^{H} \boldsymbol{G}_{k} \boldsymbol{F}
\end{aligned}
$$

where

$$
\begin{aligned}
\boldsymbol{G}_{k} & =N^{1 / 2} \operatorname{diag}\left\{g_{k}(0), g_{k}(1), \ldots, g_{k}(N-1)\right\} \\
g_{k}(i) & =N^{-1 / 2} \sum_{l=0}^{L} a_{k}(l) \exp (-j 2 \pi i l / N)
\end{aligned}
$$

and $\left[a_{k}(0), a_{k}(1), \ldots, a_{k}(L), 0, \ldots, 0\right]^{T}$ forms the first column of $\boldsymbol{A}_{k}$. Substituting Equation (27b) into Equations (17) Copyright () 2008 John Wiley \& Sons, Ltd. and (22), we have

$$
\begin{aligned}
\boldsymbol{U}_{k} & =\left(\boldsymbol{F}^{H} \boldsymbol{G}_{k}^{H}\left(\sum_{k^{\prime}=1}^{K} v_{k^{\prime}} \mathbf{G}_{k^{\prime}} \boldsymbol{G}_{k^{\prime}}^{H}+\sigma^{2} \boldsymbol{I}\right)^{-1} \boldsymbol{G}_{k} \boldsymbol{F}\right)_{\mathrm{diag}} \\
& =u_{k} \boldsymbol{I}
\end{aligned}
$$

where

$$
u_{k}=\sum_{i=0}^{N-1} \frac{\left|g_{k}(i)\right|^{2}}{N \sum_{k^{\prime}=1}^{K} v_{k^{\prime}}\left|g_{k^{\prime}}(i)\right|^{2}+\sigma^{2}}
$$

Again, substituting Equations (27b) and (29) into Equation (23) yields

$$
\begin{gathered}
\lambda_{k}=4\left(1-v_{k} u_{k}\right)^{-1}\left(\boldsymbol{F}^{H} \boldsymbol{G}_{k}^{H}\left(\sum_{k^{\prime}=1}^{K} v_{k^{\prime}} \boldsymbol{G}_{k^{\prime}} \boldsymbol{G}_{k^{\prime}}^{H}+\sigma^{2} \boldsymbol{I}\right)^{-1}\right. \\
\left.\left(\boldsymbol{Y}-\sum_{k^{\prime}=1}^{K} \boldsymbol{G}_{k^{\prime}} \boldsymbol{F} \mathrm{E}\left(\boldsymbol{x}_{k^{\prime}}\right)\right)+u_{k} \mathrm{E}\left(\boldsymbol{x}_{k}\right)\right)
\end{gathered}
$$


where

$$
\boldsymbol{Y}=\boldsymbol{F} \boldsymbol{y}
$$

Equation (31) leads to the following method to compute $\lambda_{k}$ :

Step 1. Compute $\boldsymbol{Y}-\sum_{k^{\prime}=1}^{K} \boldsymbol{G}_{k^{\prime}} \boldsymbol{F E}\left(\boldsymbol{x}_{k^{\prime}}\right)$.

Step 2. Premultiply the result of Step 1 by $\boldsymbol{G}_{k}^{H}\left(\sum_{k^{\prime}} \boldsymbol{G}_{k^{\prime}} \boldsymbol{G}_{k^{\prime}}^{H}+\sigma^{2} \boldsymbol{I}\right)^{-1}$.

Step 3. Premultiply the result of Step 2 by $\boldsymbol{F}^{H}$, add $u_{k} E\left(\boldsymbol{x}_{k}\right)$ and scale the result by $4\left(1-v_{k} u_{k}\right)^{-1}$.

Remarks:

1. The matrix inversion involved in Step 2 is trivial since $\left\{\boldsymbol{G}_{k}\right\}$ are diagonal matrices.

2. In Steps 1 and 3 , multiplying by $\boldsymbol{F}$ and $\boldsymbol{F}^{H}$ can be realised by the FFT and inverse FFT (IFFT) algorithms, respectively. The normalised complexity (in terms of operations per entry of $\boldsymbol{x}_{k}$, with each operation consisting of one multiplication and one addition) of the above algorithm is only $O\left(\log _{2} N\right)$.

3. Note that in SC-IDMA-ZP, we only need the first $J$ elements of $\lambda_{k}$.

4. In SC-IDMA-ZP, a string of zeros is involved (see Equation (26)). The means and variances of these zero elements are all set to zero in the iterative process (since they are deterministic zeros).

5. It can be shown that the detection approach for SCIDMA-CP is equivalent to that described in [21].

\subsection{Performance analysis}

An interesting feature of the above approach is that the performance of the ESE can be easily analysed. For simplicity, our discussion below is focused on the real part. The discussion for the imaginary part is similar. We can rewrite Equation (18) in a signal-plus-distortion form as

$$
\lambda_{k}^{\operatorname{Re}}(i)=\mu_{k}(i) x_{k}^{\operatorname{Re}}(i)+\zeta_{k}^{\operatorname{Re}}(i)
$$

where

$$
\begin{aligned}
\mu_{k}(i) & \equiv 4 \boldsymbol{a}_{k}(i)^{H} \boldsymbol{R}_{k}(i)^{-1} \boldsymbol{a}_{k}(i) \\
\zeta_{k}(i) & \equiv 4 \boldsymbol{a}_{k}(i)^{H} \boldsymbol{R}_{k}(i)^{-1} \\
& \left(\boldsymbol{y}-\sum_{k=1}^{K} \boldsymbol{A}_{k} \mathrm{E}\left(\boldsymbol{x}_{k}\right)-\boldsymbol{a}_{k}(i)\left(x_{k}(i)-\mathrm{E}\left(x_{k}(i)\right)\right)\right) \\
\boldsymbol{R}_{k}(i) & \equiv \sum_{k=1}^{K} v_{k} \boldsymbol{A}_{k} \boldsymbol{A}_{k}^{H}-v_{k} \boldsymbol{a}_{k}(i) \boldsymbol{a}_{k}(i)^{H}+\sigma^{2} \boldsymbol{I}
\end{aligned}
$$

Copyright (C) 2008 John Wiley \& Sons, Ltd.
It has been shown that the residue distortion at the output of the MMSE estimator [7] is approximately Gaussian distributed [18]. Since the detection approach derived above is equivalent to the MMSE approach [15], $\zeta_{k}(i)$ can be treated as a circular symmetric Gaussian random variable with zero mean and variance

$$
\mathrm{E}\left(\left|\zeta_{k}(i)\right|^{2}\right)=16 \boldsymbol{a}_{k}(i)^{H} \boldsymbol{R}_{k}(i)^{-1} \boldsymbol{a}_{k}(i)=4 \mu_{k}(i)
$$

Then, $\mathrm{E}\left(\zeta_{k}^{\operatorname{Re}}(i)^{2}\right)=2 \mu_{k}(i)$. Note that $\mu_{k}(i)$ is actually invariant to index $i$ since

$$
\begin{aligned}
\mu_{k}(i) & =4 \boldsymbol{a}_{k}(i)^{H} \boldsymbol{R}_{k}(i)^{-1} \boldsymbol{a}_{k}(i) \\
& =\frac{4 \boldsymbol{a}_{k}(i)^{H} \boldsymbol{R}^{-1} \boldsymbol{a}_{k}(i)}{1-v_{k} \boldsymbol{a}_{k}(i)^{H} \boldsymbol{R}^{-1} \boldsymbol{a}_{k}(i)} \\
& =\frac{4 u_{k}}{1-v_{k} u_{k}}
\end{aligned}
$$

where the second equality follows from the matrix inversion lemma, and the last equality follows from Equations (22) and (29). Then the ESE output $\lambda_{k}^{R e}(i)$ can be treated as an observation of $x_{k}^{R e}(i)$ scaled by $\mu_{k}(i)$ (a real constant) and corrupted by an AWGN sample $\zeta_{k}^{R e}(i)$. A single signal-tonoise-ratio (SNR) value

$$
\rho_{k} \equiv \mu_{k}(i)^{2} /\left(2 \mu_{k}(i)\right)=2 u_{k}\left(1-v_{k} u_{k}\right)^{-1}
$$

is sufficient to characterise this model. From Equation (30), $u_{k}$ is a function of $v_{k}$. Thus, Equation (37) indicates that the transfer characteristic of the ESE can be sufficiently characterised by the input average variances $\left\{v_{k}\right\}$ and the output SNRs $\rho_{k}$. Equation (37) can be used in evolution analysis to predict the system performance [16]. In particular, it facilitates the following error-floor analysis.

Suppose that the iterative detector is close to convergence so that $\left\{v_{k}\right\}$ can be treated as zeros. Then, from Equations (37) and (30), the error-floor performance of both SC schemes is determined by

$$
\sum_{i=0}^{N-1}\left|g_{k}(i)\right|^{2} /\left(\sigma^{2} / 2\right)=\sum_{l=0}^{L}\left|a_{k}(l)\right|^{2} /\left(\sigma^{2} / 2\right)
$$

The above equality holds according to Parseval's theorem. This means that the error-floor performance of both schemes only depends on the channel energy and the noise power and is independent of $N$, the number of FFT points.

The above discussion is based on the assumption of QPSK signalling with normalised energy per dimension for both ZP and CP techniques. Equation (38) indicates that, 


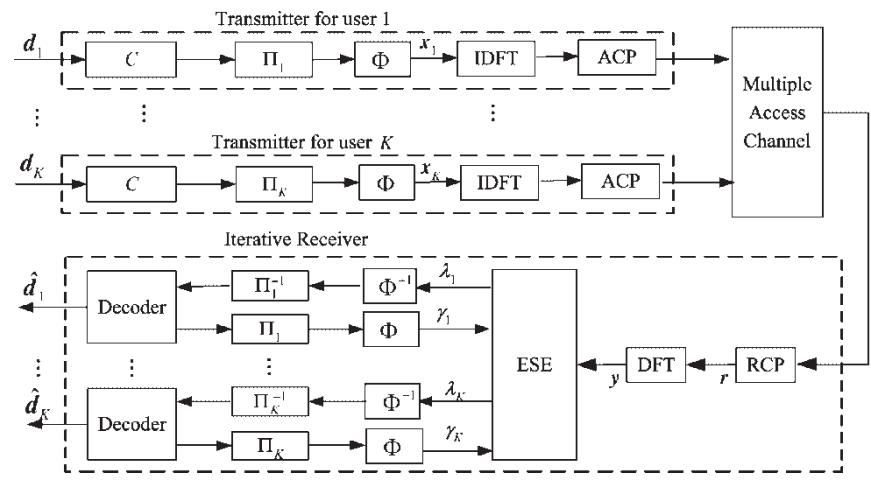

Figure 4. The ransmitter and (iterative) receiver structures of OFDM-IDMA-CP. ACP and RCP denote the operations of adding cyclic prefix and removing cyclic prefix, respectively.

given the channel energy of $\sum_{l=0}^{L}\left|a_{k}(l)\right|^{2}$ for each user, both CP- and ZP-based schemes have the same error-floor performance. However, the extra energy consumed in $\mathrm{CP}$ is not counted in the above discussion. Therefore, CP is actually less power efficient when the overhead of prefix is included. This will be verified by the simulation results in Section 5 .

\section{OFDM-IDMA SYSTEMS}

\subsection{OFDM-IDMA-CP}

We now consider the OFDM-based multi-carrier IDMA schemes. The related transceiver structure with $\mathrm{CP}$ is shown in Figure 4. This scheme has been studied in References [19] and [20]. Pre-coding using IDFT is performed at the transmitter side and pre-processing using DFT is performed at the receiver side. With $\mathrm{CP}$, the time-domain channel matrix of each user $k$ is still given by $\boldsymbol{H}_{k}^{\mathrm{cp}}$ in Equation (24). The system model is given by

$$
\boldsymbol{r}=\sum_{k=1}^{K} \boldsymbol{H}_{k}^{\mathrm{cp}} \boldsymbol{F}^{H} \boldsymbol{x}_{k}+\boldsymbol{n}
$$

The receiver applies FFT to $\boldsymbol{r}$ to obtain

$$
\boldsymbol{y}=\boldsymbol{F} \boldsymbol{r}=\sum_{k=1}^{K} \boldsymbol{A}_{k}^{\mathrm{cp}} \boldsymbol{x}_{k}+\tilde{\boldsymbol{n}}
$$

where $\boldsymbol{A}_{k}^{\mathrm{cp}}=\boldsymbol{F} \boldsymbol{H}_{k}^{c p} \boldsymbol{F}$ and $\tilde{\boldsymbol{n}}=\boldsymbol{F} \boldsymbol{n}$. Note that $\boldsymbol{n}$ and $\tilde{\boldsymbol{n}}$ have the same distribution since $\boldsymbol{F}$ is a unitary matrix.

From the property of the circulant matrix shown in Equation (27a), $\boldsymbol{A}_{k}^{\mathrm{cp}}$ is diagonal. When $K=1$, Equation (40) reduces to a standard single-user OFDM Copyright (C) 2008 John Wiley \& Sons, Ltd. system. In this case, no iteration is necessary since there is no interference among transmitted symbols. When $K>$ 1, the result in Equation (23) can again be applied to Equation (40). As $\boldsymbol{A}_{k}^{\text {cp }}$ is diagonal, $\boldsymbol{R}$ is diagonal too. The computation of $\boldsymbol{R}^{-1}$ and $\boldsymbol{U}_{k}$ is now trivial.

\subsection{OFDM-IDMA-ZP}

OFDM-IDMA-ZP can be similarly derived from its SC counterpart. IDFT is applied to the signal vector $\boldsymbol{x}_{k}$, and then the resulting vector is appended with zeros before transmission. Similarly to the channel model in Equation (26), the channel output vector can be represented as

$$
\boldsymbol{r}=\sum_{k=1}^{K} \boldsymbol{H}_{k}^{\mathrm{zp}}\left[\begin{array}{c}
\boldsymbol{F}^{H} \boldsymbol{x}_{k} \\
\mathbf{0}
\end{array}\right]+\boldsymbol{n}
$$

or equivalently,

$$
\boldsymbol{r}=\sum_{k=1}^{K} \boldsymbol{H}_{k}^{\mathrm{zp}}\left[\begin{array}{cc}
\boldsymbol{F}^{H} & \mathbf{0} \\
\mathbf{0} & \boldsymbol{I}
\end{array}\right]\left[\begin{array}{c}
\boldsymbol{x}_{k} \\
\mathbf{0}
\end{array}\right]+\boldsymbol{n}
$$

At the receiver, DFT is applied to $r$,

$$
\begin{aligned}
\boldsymbol{y} & =\tilde{\boldsymbol{F}} \boldsymbol{r}=\sum_{k=1}^{K} \tilde{\boldsymbol{F}} \boldsymbol{H}_{k}^{\mathrm{zp}}\left[\begin{array}{cc}
\boldsymbol{F}^{H} & \mathbf{0} \\
\mathbf{0} & \boldsymbol{I}
\end{array}\right]\left[\begin{array}{c}
\boldsymbol{x}_{k} \\
\mathbf{0}
\end{array}\right]+\tilde{\boldsymbol{F}} \boldsymbol{n} \\
& =\sum_{k=1}^{K} \underbrace{\tilde{\boldsymbol{G}}_{k}^{\mathrm{zp}} \tilde{\boldsymbol{F}}\left[\begin{array}{cr}
\boldsymbol{F}^{H} & \mathbf{0} \\
\mathbf{0} & \boldsymbol{I}
\end{array}\right]}_{\boldsymbol{A}_{k}}\left[\begin{array}{c}
\boldsymbol{x}_{k} \\
\mathbf{0}
\end{array}\right]+\tilde{\boldsymbol{F}} \boldsymbol{n}
\end{aligned}
$$

where

$$
\begin{aligned}
\tilde{\boldsymbol{G}}_{k}^{\mathrm{zp}} & \equiv \tilde{\boldsymbol{F}} \boldsymbol{H}_{k}^{\mathrm{zp}} \tilde{\boldsymbol{F}}^{H} \\
\boldsymbol{A}_{k} & \equiv \tilde{\boldsymbol{G}}_{k}^{\mathrm{zp}} \tilde{\boldsymbol{F}}\left[\begin{array}{cc}
\boldsymbol{F}^{H} & \mathbf{0} \\
\mathbf{0} & \boldsymbol{I}
\end{array}\right]
\end{aligned}
$$

It is important to note that the size of the DFT matrix $\tilde{\boldsymbol{F}}$ is $(J+L) \times(J+L)$, which is different from the size of the IDFT matrix $\boldsymbol{F}^{H}$ at the transmitter side. Also note that in Equation (43), $\boldsymbol{A}_{k}$ is no longer diagonal, so the system is not orthogonal even when $K=1$. Interference among different symbols from the same user is inevitable in OFDM-IDMA-ZP.

The result in Equation (23) can again be applied to Equation (43) which can be efficiently implemented as 
follows. Note that

$$
\begin{aligned}
\boldsymbol{A}_{k} \boldsymbol{A}_{k}^{H} & =\tilde{\boldsymbol{G}}_{k}^{\mathrm{zp}} \tilde{\boldsymbol{F}}\left[\begin{array}{cc}
\boldsymbol{F}^{H} & \mathbf{0} \\
\mathbf{0} & \boldsymbol{I}
\end{array}\right]\left[\begin{array}{ll}
\boldsymbol{F} & \mathbf{0} \\
\mathbf{0} & \boldsymbol{I}
\end{array}\right] \tilde{\boldsymbol{F}}^{H}\left(\tilde{\boldsymbol{G}}_{k}^{\mathrm{zp}}\right)^{H} \\
& =\tilde{\boldsymbol{G}}_{k}^{\mathrm{zp}}\left(\tilde{\boldsymbol{G}}_{k}^{\mathrm{zp}}\right)^{H}
\end{aligned}
$$

is diagonal. Thus, the covariance matrix (see Equation (17))

$$
\boldsymbol{R}=\sum_{k=1}^{K} v_{k} \tilde{\boldsymbol{G}}_{k}^{\mathrm{zp}}\left(\tilde{\boldsymbol{G}}_{k}^{\mathrm{zp}}\right)^{\mathrm{H}}+\sigma^{2} \boldsymbol{I}
$$

is also diagonal and so its inversion is trivial. In what follows, we will show how to compute $\boldsymbol{U}_{k}$ efficiently. Let $\boldsymbol{M}=\tilde{\boldsymbol{F}}^{H}\left(\tilde{\boldsymbol{G}}_{k}^{\mathrm{zp}}\right)^{H} \boldsymbol{R}^{-1} \tilde{\boldsymbol{G}}_{k}^{\mathrm{zp}} \tilde{\boldsymbol{F}}$ that is circulant since $\left(\tilde{\boldsymbol{G}}_{k}^{\mathrm{zp}}\right)^{H} \boldsymbol{R}^{-1} \tilde{\boldsymbol{G}}_{k}^{\mathrm{zp}}$ is diagonal. It can be shown that $u_{k}^{\mathrm{zp}}(i)$, the $i$ th diagonal entry of $\boldsymbol{U}_{k}=\left(\boldsymbol{A}_{k}^{H} \boldsymbol{R}^{-1} \boldsymbol{A}_{k}\right)_{\text {diag }}$, is given by

$$
u_{k}^{\mathrm{zp}}(i)=\boldsymbol{e}_{i}^{T}\left[\begin{array}{cc}
\boldsymbol{F} & \mathbf{0} \\
\mathbf{0} & \boldsymbol{I}
\end{array}\right] \boldsymbol{M}\left[\begin{array}{cc}
\boldsymbol{F}^{H} & \mathbf{0} \\
\mathbf{0} & \boldsymbol{I}
\end{array}\right] \boldsymbol{e}_{i}
$$

where $\boldsymbol{e}_{i}$ is the $i$ th column of an identity matrix with proper size. Note that we only need the first $J$ diagonal elements. We can rewrite Equation (47) as

$$
u_{k}^{\mathrm{zp}}(i)=\boldsymbol{f}_{i}^{H} \widehat{\boldsymbol{M}} \boldsymbol{f}_{i}
$$

where matrix $\widehat{\boldsymbol{M}}$ (of size $J \times J)$ is the upper-left part of $\boldsymbol{M}$ (of size $(J+L) \times(J+L)$ ), and $\boldsymbol{f}_{i}$ the $i$ th column of $\boldsymbol{F}^{H}$. It can be verified that $\widehat{\boldsymbol{M}}$ can be expressed in the following Toeplitz form,

$$
\widetilde{\boldsymbol{M}}=\left[\begin{array}{ccccc}
\alpha_{0} & \alpha_{1} & \cdots & \alpha_{J-2} & \alpha_{J-1} \\
\alpha_{-1} & \alpha_{0} & \alpha_{1} & \ddots & \alpha_{J-2} \\
\vdots & \alpha_{-1} & \alpha_{0} & \ddots & \vdots \\
\alpha_{-J+2} & \ddots & \ddots & \ddots & \alpha_{1} \\
\alpha_{-J+1} & \alpha_{-J+2} & \cdots & \alpha_{-1} & \alpha_{0}
\end{array}\right]
$$

Hence,

$$
\begin{aligned}
\boldsymbol{f}_{i}^{H} \widehat{\boldsymbol{M}} \boldsymbol{f}_{i}= & \alpha_{0}+\frac{(J-1) \alpha_{-1}+\alpha_{J-1}}{J} e^{-j \frac{2 \pi i}{J}} \\
& +\frac{(J-2) \alpha_{-2}+2 \alpha_{J-2}}{J} e^{-j \frac{2 \pi \cdot 2 i}{J}}
\end{aligned}
$$

Copyright (C) 2008 John Wiley \& Sons, Ltd.

$$
\begin{aligned}
& +\frac{(J-3) \alpha_{-3}+3 \alpha_{J-3}}{J} e^{-j \frac{2 \pi \cdot 3 i}{J}}+\cdots \\
= & \sum_{n=0}^{J-1} \frac{(J-n) \alpha_{-n}+n \alpha_{J-n}}{J} e^{-j \frac{2 \pi n i}{J}}
\end{aligned}
$$

In the above equation, $\alpha_{J}=0$. Thus, $\left\{u_{k}^{\mathrm{zp}}(i)\right\}$ can be efficiently calculated by taking the FFT of $\left\{J^{-1}((J-\right.$ $\left.\left.n) \alpha_{-n}\right)+n \alpha_{J-n}\right\}$. Due to the extra IFFT and FFT operations, the cost of the OFDM-IDMA-ZP approach is higher than that of SC-IDMA-ZP.

Performance analysis of both OFDM-IDMA-CP and OFDM-IDMA-ZP is complicated owing to the frequencyselective nature of the OFDM scheme involved. We are still working on this issue at the moment and so for present purposes we will rely on simulation results for performance evaluation.

\section{NUMERICAL RESULTS}

In this section, numerical results are provided to compare the performance of the previously discussed schemes. We first introduce some common settings used in the simulations. All of the schemes (SC-IDMA-CP, SCIDMA-ZP, OFDM-IDMA-CP and OFDM-IDMA-ZP) employ the same encoding scheme: a rate-1/2 convolution code with generator $(23,35)_{8}$, followed by length-16 repetition code. The data length for each user is 512 . QPSK modulation is used. The number of users is fixed at 32 . The block size $J$ for the four schemes is 64 . The channel memory length $L$ is 16 , that is $L=J / 4$. These parameter values correspond to a relatively high speed environment where channel conditions change rapidly. The transmitted block in such an environment cannot be too long and so the guard interval represents a considerable overhead. We assume independent block fading channels. For each block, the samples of the channel taps for each user are independently drawn from a complex Gaussian distribution with mean 0 and variance $1 /(L+1)$ (i.e. the average channel energy is normalised to 1$)$.

The performance of SC-IDMA-CP and SC-IDMA-ZP with different numbers of iterations is shown in Figure 5. The extra energy required by the $\mathrm{CP}$ approach is included in $E_{b} / N_{0}$. We can see that, in the high BER range (waterfall range), the performance of SC-IDMA-ZP is significantly better than that of SC-IDMA-CP. In the low BER range (error-floor range), for example at $\mathrm{BER}=1 \times 10^{-5}$ with 15 iterations, the performance gap between SC-IDMA$\mathrm{CP}$ and SC-IDMA-ZP is about $1 \mathrm{~dB}$, which matches the 


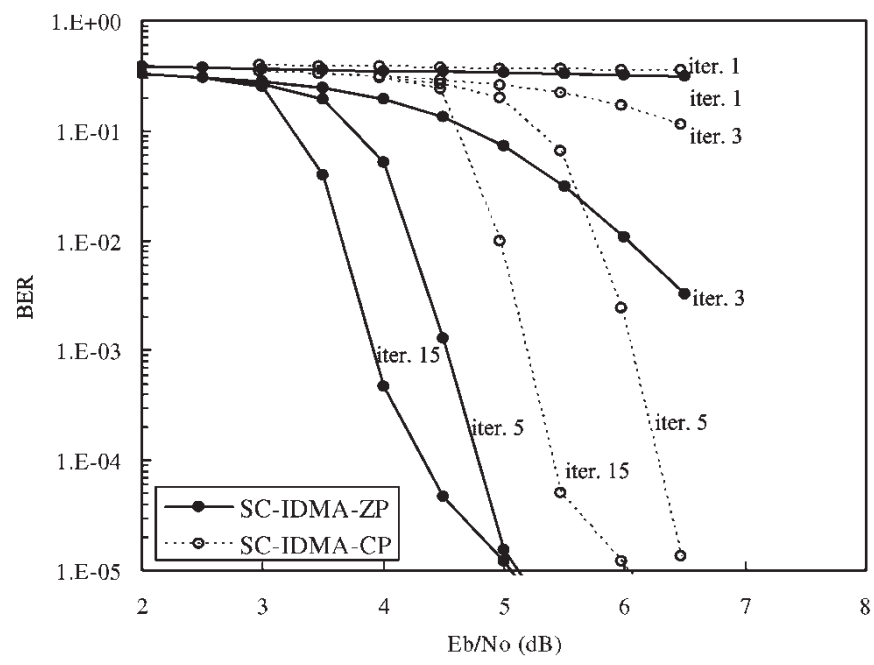

Figure 5. Performance comparison between SC-IDMA-CP and SC-IDMA-ZP.

extra energy consumed by CP (i.e. $10 \log ((64+16) / 64)=$ $0.9691 \mathrm{~dB})$.

Figure 6 shows the performance of OFDM-IDMA-CP and OFDM-IDMA-ZP. We can see that OFDM-IDMA-ZP outperforms OFDM-IDMA-CP by about $1 \mathrm{~dB}$ in error-floor range, which again matches the extra energy consumed by $\mathrm{CP}$.

From Figures 5 and 6, we can also observe that the convergence of the ZP-based schemes is faster than that of the CP-based schemes. This attributes to the spreading effect introduced by the multipath channel as discussed in Subsection 3.2.

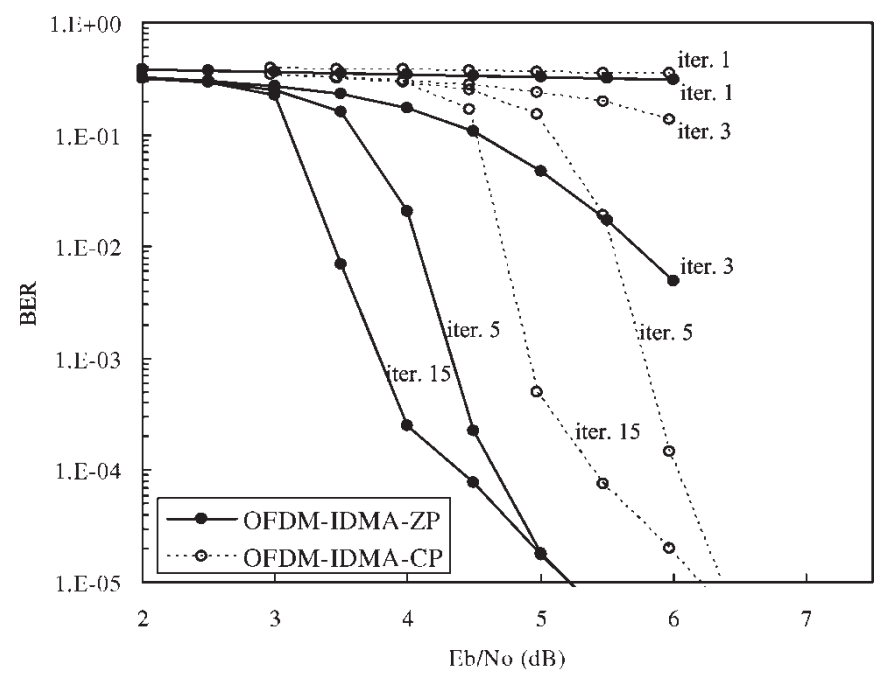

Figure 6. Performance comparison between OFDM-IDMA-ZP and OFDM-IDMA-CP.

Copyright (C) 2008 John Wiley \& Sons, Ltd.

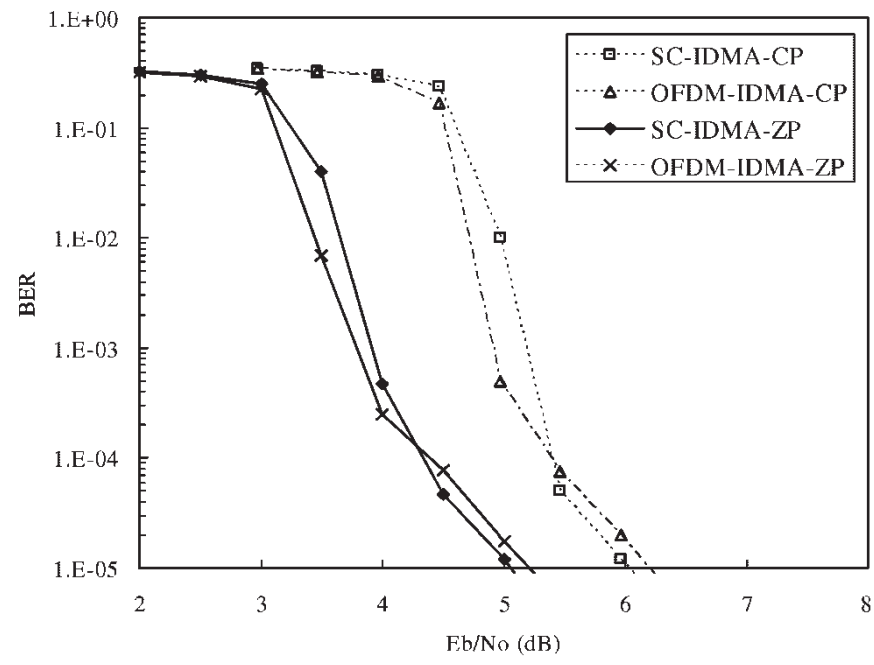

Figure 7. Performance of the four schemes. The number of iterations for all the schemes is 15 .

In Figure 7, we compare the CP- and ZP-based schemes together. We can see that OFDM-IDMA-CP and SC-IDMA-CP have similar performance, and so do SC-IDMA-ZP and OFDM-IDMA-ZP. The ZP-based schemes are generally more power efficient.

\section{CONCLUSIONS}

In this paper, we have investigated single- and multi-carrier IDMA schemes with CP and ZP techniques. We have outlined a generic detection principle that can be applied to these schemes. Fast implementation techniques have been developed by exploiting the circulant property of the channel matrices involved. We have also demonstrated the advantages of ZP-based schemes in power efficiency and convergence speed.

\section{ACKNOWLEDEGMENT}

This work was fully supported by a grant from the Research Grant Council of the Hong Kong SAR, China [Project No. CityU 116706].

\section{REFERENCES}

1. Hanzo L, Münster M, Choi BJ, Keller T. OFDM and MC-CDMA for Broadband Multi-User Communications. WLANs and Broadcasting, John Wiley Sons Chichester, UK, 2003.

2. Fazel K, Kaiser S. Multi-Carrier and Spread Spectrum Systems. John Wiley Sons, Chichester: UK, 2003.

Eur. Trans. Telecomms. 2008; 19:537-547

DOI: $10.1002 /$ ett 
3. Falconer D, Ariyavisitakul SL. Benyamin-Seeyar A, Eldson B. Frequency domain equalization for single-carrier broadband wireless systems. IEEE Communications Magazine 2002; 58-66.

4. Muquet B, de Courville M, Duhamel P, Giannakis GB, Magniez P. Turbo demodulation of zero-padded OFDM transmissions. IEEE Transactions on Communications 2002; 50(11):1725-1728.

5. Wang Z, Ma X, Giannakis GB. OFDM or single-carrier block transmissions? IEEE Transactions on Communications 2004; 52(3):380-394.

6. Muquet B, Wang Z, Giannakis GB, de Courville M, Duhamel P. Cyclic prefixing or zero padding for wireless multicarrier transmissions? IEEE Transactions on Communications 2002; 50(12):2136-2148.

7. Wang X, Poor HV. Iterative (turbo) soft interference cancellation and decoding for coded CDMA. IEEE Transactions on Communications 1999; 47:1046-1061.

8. Tüchler M, Koetter R, Singer AC. Turbo equalization: principles and new results. IEEE Transactions on Communications 2002; 50:754767.

9. Wang P, Xiao J, Ping L. Comparison of orthogonal and non-orthogonal approaches to future wireless cellular systems. IEEE Vehicular Technology Magazine 2006; 1:4-11.

10. 3rd Generation Partnership project, Technical Specification Group Radio Access Network, Physical Layer Aspects for Evolved UTRA (Release 7), 3GPP TR25.814 V1.0.1 (2005-11).

11. Ping L, Liu L, Wu K, Leung WK. Interleave division multiple-access. IEEE Transactions on Wireless Communications 2006; 5(4):938-947.

12. Schoeneich $\mathrm{H}$, Hoeher PA. Adaptive interleave division multiple access - a potential air interface for $4 \mathrm{G}$ bearer services and wireless LANs. In Proceedings of WOCN 2004, Muscat, Oman, June 2004, pp. $179-182$.
13. Berrou C, Glavieux A, Thitimajshima P. Near Shannon limit errorcorrecting coding and decoding: turbo codes. In Proceedings of IEEE International Conference on Communications, Geneva, Switzerland, May 1993.

14. Liu L, Leung WK, Ping L. Simple chip-by-chip multiuser detection for CDMA systems. IEEE Vehicular Technology Conference, VTC'03, pp. 2157-2161.

15. Yuan X, Wu K, Ping L. The jointly Gaussian approach to iterative detection in MIMO Systems. In Proceedings of IEEE International Conference on Communication, ICC'06, Istanbul, Turkey, 11-15 June 2006.

16. Yuan X, Guo Q, Ping L. Evolution analysis of iterative LMMSE-APP detection for coded linear system with cyclic prefixes. In Proceedings of ISIT 2007, Nice, France, June 2007.

17. Horn RA, Johnson CR. Matrix Analysis. Cambridge University Press Cambridge, UK, 1990.

18. Poor HV, Verdu S. Probability of error in MMSE multiuser detection. IEEE Transactions on Information Theory 1997; 43:835-847.

19. Mahafeno I, Langlais C, Jego C. OFDM-IDMA versus IDMA with ISI cancellation for quasi-static Rayleigh fading multipath channels. In Proceedings of 4th International Symposium Turbo Codes Related Topics, Munich, Germany, April 3-7, 2006.

20. Zhou S, Li Y, Zhao M, Xu X, Wang J, Yao Y. Novel techniques to improve downlink multiple access capacity for beyond 3G. IEEE Communication Magazine 2005; 43:61-69.

21. Guo Q, Yuan X, Ping L. Multi-user detection techniques for potential 3GPP long term evolution (LTE) schemes. In Proceedings of 6th international workshop on multi-carrier spread spectrum, Herrsching, Germany, 7-9 May 2007. pp. 77-86. 